\title{
A Wrapping Method for Inserting Titanium Micro- Mesh Implants in the Reconstruction of Blowout Fractures
}

\author{
Tae Joon Choi, Jin Sik Burm, Won Yong Yang, Sang Yoon Kang \\ Department of Plastic and Reconstructive Surgery, Kyung Hee University School of Medicine, Seoul, Korea
}

Titanium micro-mesh implants are widely used in orbital wall reconstructions because they have several advantageous characteristics. However, the rough and irregular marginal spurs of the cut edges of the titanium mesh sheet impede the efficacious and minimally traumatic insertion of the implant, because these spurs may catch or hook the orbital soft tissue, skin, or conjunctiva during the insertion procedure. In order to prevent this problem, we developed an easy method of inserting a titanium micro-mesh, in which it is wrapped with the aseptic transparent plastic film that is used to pack surgical instruments or is attached to one side of the inner suture package. Fifty-four patients underwent orbital wall reconstruction using a transconjunctival or transcutaneous approach. The wrapped implant was easily inserted without catching or injuring the orbital soft tissue, skin, or conjunctiva. In most cases, the implant was inserted in one attempt. Postoperative computed tomographic scans showed excellent placement of the titanium micro-mesh and adequate anatomic reconstruction of the orbital walls. This wrapping insertion method may be useful for making the insertion of titanium micro-mesh implants in the reconstruction of orbital wall fractures easier and less traumatic.

Keywords: Orbital fracture / Titanium / Surgical mesh / Procedure, Reconstructive surgical

\author{
Correspondence: Jin Sik Burm \\ Department of Plastic and \\ Reconstructive Surgery, Kyung Hee \\ University School of Medicine, \\ 23 Kyungheedae-ro, Dongdaemun-gu, \\ Seoul 02447, Korea \\ Tel: +82-2-958-8431 \\ Fax: +82-2-963-5638 \\ E-mail: jsburm@gmail.com
}

This article was presented as a poster at the 71th Congress of the Korean Society of Plastic and Reconstructive Surgeons on November 1-3, 2013, in Seoul, Korea.

No potential conflict of interest relevant to this article was reported.

Received: 20 Jul 2015 • Revised: 20 Aug $2015 \bullet$ Accepted: 27 Aug 2015

pISSN: 2234-6163 • elSSN: 2234-6171 • http://dx.doi.org/10.5999/aps.2016.43.1.84 • Arch Plast Surg 2016;43:84-87

\section{INTRODUCTION}

Previous studies have shown that titanium micro-mesh implants are valuable in the accurate three-dimensional anatomic reconstruction of orbital blowout fractures $[1,2]$. Titanium micromesh implants are thin, easily bent according to the configuration of the defect, and do not have shape memory. They have additional advantages, such as good physical strength, the ability to be easily trimmed, good drainage, high corrosion resistance, rapid tissue ingrowth into their pores, visibility on radiographs, a low infection rate, excellent biocompatibility, and low cost [1-
4]. However, rough and irregular marginal spurs are inevitably formed along the cut edges of the titanium micro-mesh during trimming (Fig. 1). Furthermore, they tend to catch adjacent soft tissue during implant insertion, impeding accurate and minimally traumatic implant insertion $[5,6]$ and potentially resulting in malposition and injury to the orbital skin, conjunctiva, or orbital tissues. This difficulty in insertion may be a key disadvantage of titanium micro-mesh implants, and could lead surgeons to use alternative materials, such as absorbable meshes. In order to solve this problem, we developed an easy wrapping insertion method that uses an aseptic transparent plastic film. 


\section{IDEA}

In the operations described in this study, we usually used a 0.1 mm-thick micro-titanium augmentation mesh (M-TAM, Stryk-

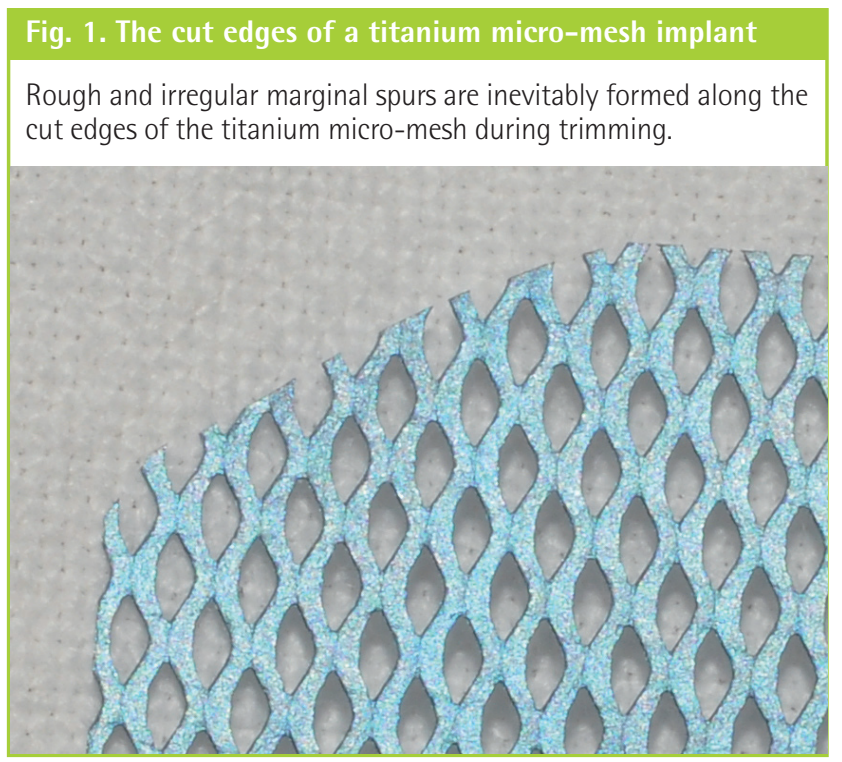

er Leibinger GmbH, Freiburg im Breisgau, Germany) to reconstruct the orbital wall in orbital blowout fractures. When the orbital bony defect was so large that a $0.1 \mathrm{~mm}$-thick titanium micro-mesh could not support sufficiently to the great amount of herniated orbital tissues, we used a $0.2 \mathrm{~mm}$-thick titanium micro-mesh. After the bony defect of the orbital wall was fully exposed, a titanium micro-mesh sheet was trimmed with scissors and molded depending on the size and shape of the bony defect. An aseptic transparent plastic film was harvested from one side of the inner package of a black silk or nylon suture (Ailee Co., Busan, Korea) or from one side of a sterilized laminated film pouch that is used to pack a small surgical instrument. The film was trimmed with scissors to approximately $4 \times 5 \mathrm{~cm}$. The trimmed micro-mesh sheet was wrapped in the plastic film circumferentially, keeping both ends of the film overlapped at the midline. Thus, the micro-mesh sheet was completely enveloped without any exposure (Fig. 2A). The titanium mesh and plastic film were gripped together using forceps. While both endings of the incisional opening and the orbital contents were held with small retractors and a malleable retractor, the wrapped implant was inserted into the orbital cavity through the incisional open-

\section{Fig. 2. Wrapping insertion of titanium micro-mesh implants}

(A) An ultrathin titanium micro-mesh implant wrapped with a transparent plastic film. (B) Insertion of the wrapped implant through an incision for the inferior orbital wall and (C) the medial orbital wall. (D) Pulling out the film with a mosquito hemostat while grasping the inserted implant with a forceps.
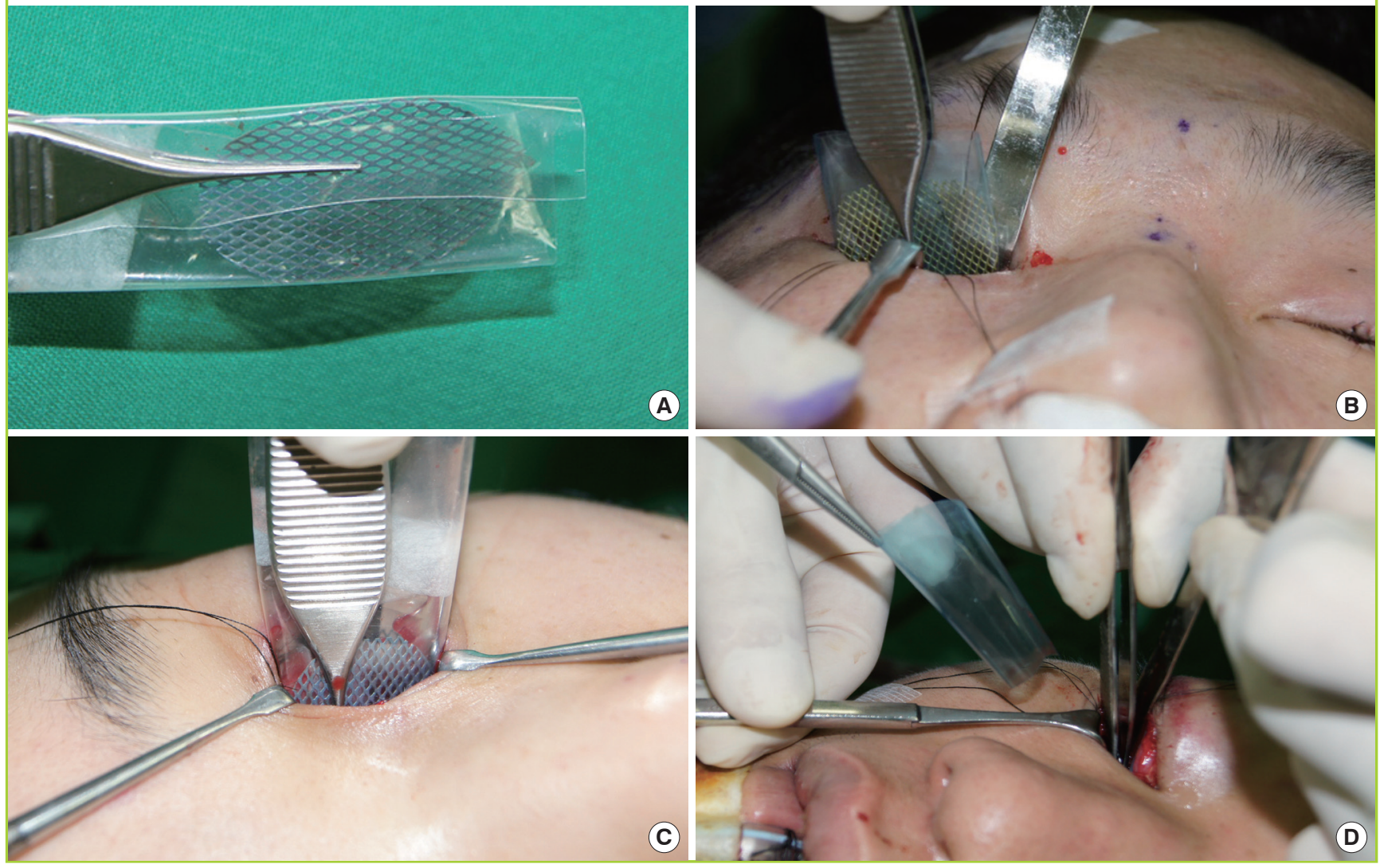


\section{Fig. 3. Postoperative computed tomography scans}

(A) A postoperative computed tomography scan showed excellent placement of the titanium micro-mesh (yellow arrow) and anatomic reconstruction of the inferior orbital wall and (B) the medial orbital wall.

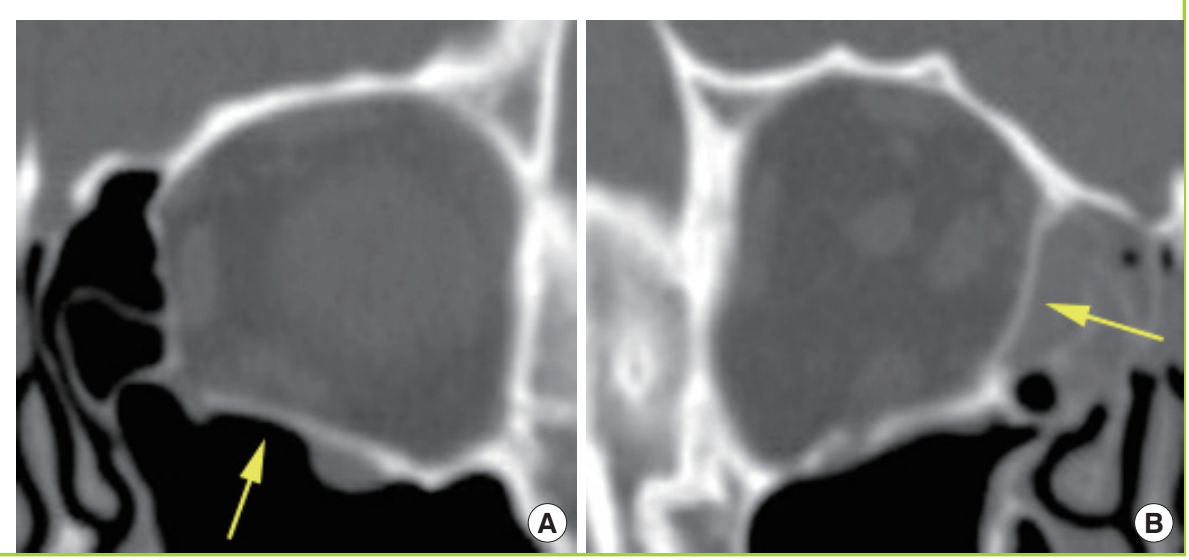

ing and placed into the desired position, without hooking or catching the orbital soft tissue (Fig. 2B, C). After an additional reset of the implant into its final position, the film was gently pulled out with a mosquito hemostat while grasping the inserted implant with a forceps (Fig. 2D).

Fifty-four patients underwent orbital wall reconstruction with titanium micro-mesh implants. The fractured orbital wall was exposed by the transcutaneous or transconjunctival approach. The average size of the titanium mesh implant was $2.1 \times 2.0 \mathrm{~cm}$ (maximum, $2.5 \times 2.8 \mathrm{~cm}$ ) in inferior orbital wall reconstruction, and $2.9 \times 2.2 \mathrm{~cm}$ (maximum, $3 \times 2.5 \mathrm{~cm}$ ) in medial orbital wall reconstruction. We were able to easily insert large implants through the incision and adjust them to their desired position in the orbital cavity without hooking the skin, conjunctiva, or orbital tissue, due to the smooth surface of the plastic film. No injuries to the skin, conjunctiva, or orbital soft tissue occurred. In most cases, the titanium micro-mesh implant was inserted in one attempt. In one case, the film was torn by the marginal spurs of the titanium mesh while it was being pulled out. However, even in that case, no fragments of the film remained in situ. Postoperative computed tomographic scans showed excellent placement of the titanium micro-mesh and anatomic reconstruction of the orbital wall (Fig. 3).

\section{DISCUSSION}

Various synthetic materials are widely used as alloplastic materials for orbital wall fracture repair, such as porous polyethylene sheets, absorbable mesh, and titanium mesh. Ultrathin titanium micro-mesh has several advantageous characteristics, such as good physical strength, the ability to be easily trimmed and molded, good drainage, high corrosion resistance, tissue ingrowth into its pores, visibility on radiographs, a low infection rate, and excellent biocompatibility in terms of osseointegration and tissue incorporation, which make it a valuable material for orbital wall reconstruction [1-4].

Nonetheless, absorbable meshes are commonly used for orbital wall reconstruction [7]. This tendency is most likely due to the sharp points and irregular marginal spurs along the cut edges of the titanium micro-mesh, which tend to catch or hook adjacent soft tissue, making placement difficult [5,6]. Sharp and irregular marginal spurs of the cut edges are inevitably formed during trimming. Eliminating all sharp points is very time-consuming and impossible to carry out completely due to the structure of the mesh. As well as impeding accurate and minimally traumatic implant insertion, the titanium micro-mesh sometimes results in injuries to the skin, conjunctiva, and orbital tissue. The orbital cavity contains several vulnerable vital structures [8]. Diplopia can even occur when a vital structure, such as an extraocular muscle, is injured or entrapped [9].

In order to solve these problems, we developed this wrapping insertion method. It has several benefits. First, it facilitates the smooth and less traumatic insertion and placement of the titanium micro-mesh. Efficacious and smooth implant insertion makes accurate placement easier and shortens the operation time. Less trauma to the orbital tissues results in fewer complications such as postoperative diplopia, swelling, hemorrhage, scarring, and consequent adhesion and restriction of ocular movement. Sometimes a mesh is repetitively moved into and out of the orbital cavity until the proper contour and size are achieved. Using our technique, this process can be performed easily without injury to the skin, conjunctiva, and orbital contents. Second, the incision length can be shortened because of the decreased fear of injury to the skin and orbital tissue. Third, this method is extremely practical. In most medical institutions, it is easy to obtain an aseptic transparent plastic film from inner suture packages or the sterilized pouches of surgical instruments during an operation without any additional cost and effort. This 
film is smooth and is not easily torn. It causes no visual disturbance during deployment of the implant because it is transparent. Thus, we suggest that this plastic film is an excellent material for surgeons to use.

Since we developed this wrapping insertion method, it has been routinely used in orbital wall reconstruction using titanium micro-mesh implants. We believe that it is a very useful surgical technique that makes the reconstruction of orbital wall fractures using titanium micro-mesh implants easier and safer.

\section{REFERENCES}

1. Sugar AW, Kuriakose M, Walshaw ND. Titanium mesh in orbital wall reconstruction. Int J Oral Maxillofac Surg 1992; 21:140-4.

2. Park HS, Kim YK, Yoon CH. Various applications of titanium mesh screen implant to orbital wall fractures. J Craniofac Surg 2001; 12:555-60.

3. Kuttenberger JJ, Hardt N. Long-term results following re- construction of craniofacial defects with titanium micromesh systems. J Craniomaxillofac Surg 2001;29:75-81.

4. Schubert W, Gear AJ, Lee C, et al. Incorporation of titanium mesh in orbital and midface reconstruction. Plast Reconstr Surg 2002;110:1022-30.

5. Tabrizi R, Ozkan TB, Mohammadinejad C, et al. Orbital floor reconstruction. J Craniofac Surg 2010;21:1142-6.

6. Gabrielli MF, Monnazzi MS, Passeri LA, et al. Orbital wall reconstruction with titanium mesh: retrospective study of 24 patients. Craniomaxillofac Trauma Reconstr 2011;4:1516.

7. Moon SJ, Suh HS, Park BY, et al. Safety of silastic sheet for orbital wall reconstruction. Arch Plast Surg 2014;41:362-5.

8. Chang TY, Lee JW. An innovative technique in orbital floor reconstruction avoiding complications: temporary use of the silicone guide. Formosan J Surg 2014;47:99-104.

9. Mauriello JA Jr. Inferior rectus muscle entrapped by Teflon implant after orbital floor fracture repair. Ophthal Plast Reconstr Surg 1990;6:218-20. 